\title{
(6) OPEN ACCESS \\ Service innovation in glaucoma management: using a web-based electronic patient record to facilitate virtual specialist supervision of a shared care glaucoma programme
}

\author{
Heathcote R Wright, ${ }^{1}$ Jeremy P Diamond ${ }^{2}$
}

${ }^{1}$ Centre for Eye Research Australia, Royal Victorian Eye \& Ear Hospital, East Melbourne, Victoria, Australia ${ }^{2}$ Bristol Eye Hospital, Bristol, UK

\section{Correspondence to} Dr Heathcote R Wright, Centre for Eye Research Australia, Royal Victorian Eye \& Ear Hospital, Peter Howson Wing Level 1, 32 Gisborne Street, East Melbourne, VIC 3002, Australia; heathcote27@gmail. com

Received 4 June 2014 Revised 12 September 2014 Accepted 13 September 2014 Published Online First 21 October 2014

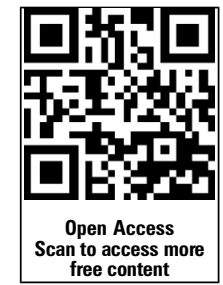

CrossMark

\section{To cite: Wright $\mathrm{HR}$,}

Diamond JP. Br J

Ophthalmol 2015;99:

313-317.

\begin{abstract}
Aim To assess the importance of specialist supervision in a new model of glaucoma service delivery. Methods An optometrist supported by three technicians managed each glaucoma clinic. Patients underwent testing and clinical examination before the optometrist triaged them into one of five groups: 'normal', 'stable', 'low risk', 'unstable' and 'high risk'. Patient data were uploaded to an electronic medical record to facilitate virtual review by a glaucoma specialist.
\end{abstract}

Results 24257 glaucoma reviews at three glaucoma clinics during a 31-month period were analysed. The clinic optometrists and glaucoma specialists had substantial agreement ( $\kappa$ 0.69). 13 patients were identified to be high risk by the glaucoma specialist that had not been identified as such by the optometrist. Glaucoma specialists amended $13 \%$ of the optometrists' interim decisions resulting in an overall reduction in review appointments by $2.4 \%$.

Conclusions Employing technicians and optometrists to triage glaucoma patients into groups defined by risk of blindness allows higher risk patients to be directed to a glaucoma specialist. Virtual review allows the glaucoma specialist to remain in overall control while reducing the risk that patients are treated or followed-up unnecessarily. Demand for glaucoma appointments can be reduced allowing scarce medical resources to be directed to patients most in need.

\section{INTRODUCTION}

The United Kingdom Hospital Eye Service (HES) is responsible for diagnosing and managing glaucoma including ocular hypertension and glaucomasuspects. Due to an ageing population, earlier detection of cases and new referral recommendations from the National Institute for Health and Care Excellence, ${ }^{1}$ there has been a dramatic increase in the number of patients requiring review. This is a pattern that will further increase as the population ages. There are about 1.2 million glaucoma patients in England who require approximately 2.4 million review appointments per annum. ${ }^{2}$ An additional 500000 patients are likely to be brought into the system within the next few years (JM Sparrow, personal communication, 2011).

HES glaucoma departments are struggling to review glaucoma patients at their planned interval, as demonstrated by an alert from the National Patient Safety Association, suggesting that patients have come to harm because their glaucoma review appointments were delayed. ${ }^{3}$ In an attempt to cope with the increasing workload, several HES glaucoma departments have outsourced review of their patients to community optometrists. ${ }^{4}$ Appropriately trained optometrists have previously been shown to safely assess glaucoma patients. ${ }^{5-7}$ Three potential concerns with such a system are: (1) failure to identify at-risk patients who require urgent attention for life or sight threatening complications, (2) overtreatment of patients who do not have glaucoma and (3) there is anecdotal evidence that optometrists may have a more conservative approach to review appointments; which could result in overservicing of patients and an increase in demand on an already stretched service. We look at the role of glaucoma specialist virtual-supervision of communitybased optometrists.

In summer 2007, the Bristol Eye Hospital (BEH) invited a private company (Newmedica) to review a backlog of 4000 glaucoma patients who were overdue for their review appointment (by up to 2 years). Newmedica provided optometrists, technicians, equipment and support infrastructure. Patients were recruited from the general follow-up pool and were not 'cherry-picked' to exclude complex patients. All clinical data including history, examination findings, visual fields and colour optic disc images were uploaded to a web-based electronic patient record (EPR) (EMMA, New Medical Systems, London, UK). The clinic was initially run within the $\mathrm{BEH}$ precinct but was subsequently re-engineered to run from a mobile unit sited in the community. The clinic continued to function after the initial 4000 patients were seen and additional clinics were established in other centres including Nuneaton and Kingston.

The optometrist assessing the patient categorised them according to a five-step glaucoma management algorithm. The algorithm was designed after consultation between three senior glaucoma consultants to differentiate between patients with glaucoma of varying degrees of severity (figure 1):

1. 'Normal', with no evidence of glaucoma (to be discharged).

2. 'Stable', glaucoma with a low risk of lifetime blindness (to be reviewed in 12 months).

3. 'Low risk', stable glaucoma with a moderate risk of lifetime blindness (to be reviewed 6 months).

4. 'Unstable' glaucoma (requiring prompt evaluation by a glaucoma specialist within 6 weeks). 

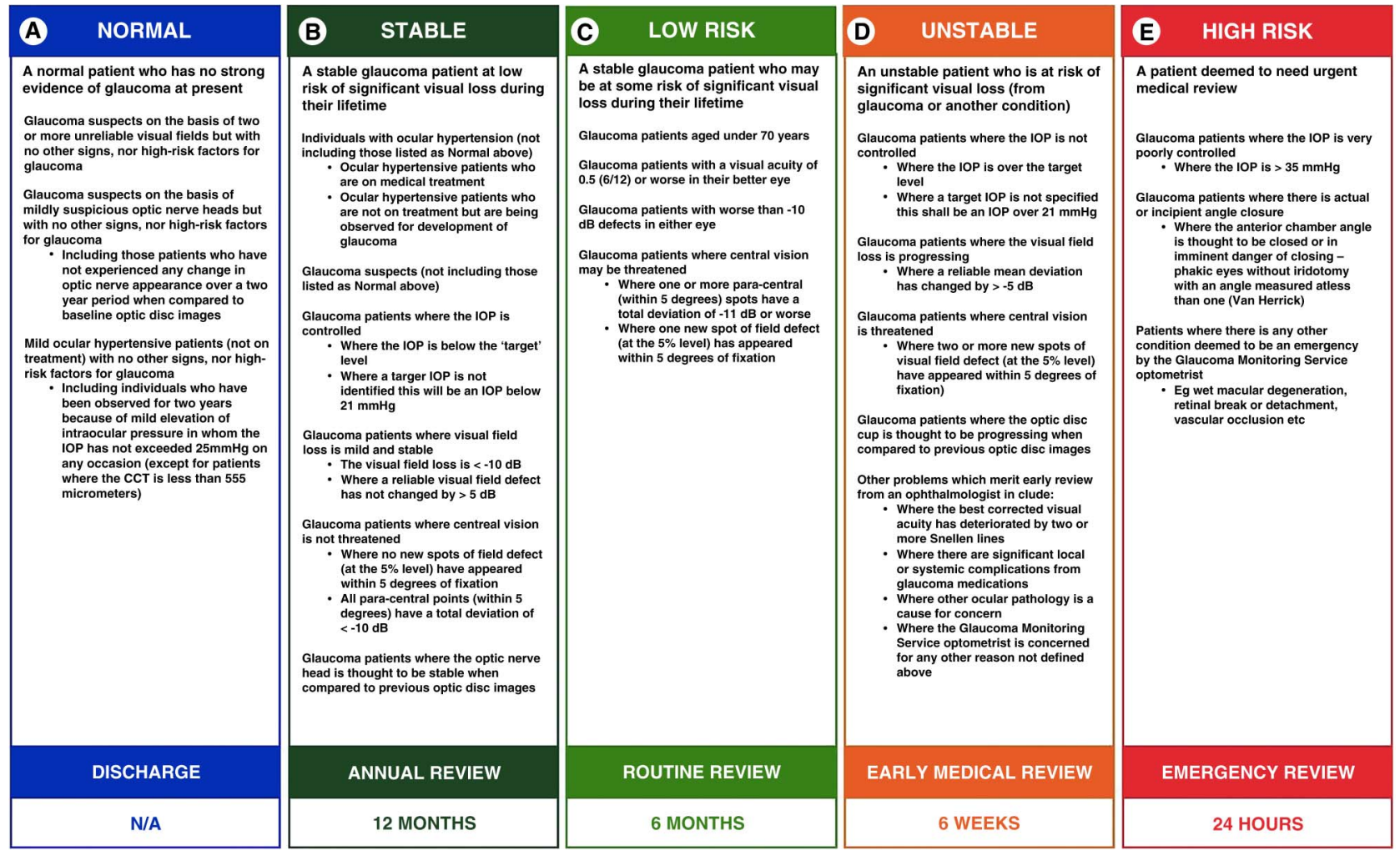

Figure 1 Five-step glaucoma management algorithm.

5. 'High-risk' glaucoma (requiring urgent assessment by a glaucoma specialist within $24 \mathrm{~h}$ ).

The clinical information was uploaded to a web-based EPR. Within 1 week of the clinic appointment, a glaucoma specialist would perform a virtual review by accessing the EPR over the internet. Patients were advised that the interim outcome might change after a glaucoma specialist reviewed their clinical data. After reviewing all the clinical data, the specialist would either confirm the optometrists' interim outcome or change it if clinically indicated (Figure 2).

Virtual review represents a new model of care in which optometrists manage patients within the community and receive online supervision from glaucoma specialists. We assessed the correlation between optometrist and glaucoma specialist to determine whether this extra level of review is required. During development of the model, it was argued that glaucoma specialist supervision would be important in two main areas. (1) Safety: by identifying high-risk patients who might be missed by the optometrist. (2) Efficiency: by re-evaluating the diagnostic category of a patient and decreasing the number of unnecessary review appointments generated. We evaluated data collected over a 31-month period to determine if this was indeed the case.

\section{MATERIALS AND METHODS}

We collected data from 1 September 2010 to 28 March 2013 from Bristol, Nuneaton and Kingston. For all visits to the mobile clinic optometrist during this period, the category assigned by the optometrist and that assigned by the glaucoma specialist in the virtual review clinic were entered into SPSS V.21. Ophthalmologists were aware of the category assigned by the optometrist when they made their decision. Mobile clinical units would generally see about 20 patients in a half-day session. Ophthalmologists review electronic records at a rate of approximately 15-20 per hour.

Kappa coefficient was used to measure inter-observer agreement between the category assigned by the optometrist and that assigned by the glaucoma specialist. All patients who were considered high risk were assessed to determine if there was a safety issue with optometrists missing potential sight threatening cases. Finally, we compared the number of visits generated by optometrists grading as opposed to the number of visits generated by glaucoma specialist grading. This was an audit of a programme that is currently delivering routine patient care. No identifiable patient data were collected; therefore, ethics approval was not required.

\section{RESULTS}

Data were analysed for all patients seen in clinics in Bristol, Nuneaton and Kingston during the study period. A total of 24257 patient assessments were performed. The category assigned by the optometrist and that assigned by the glaucoma specialist are shown in table 1 . The kappa statistic for interobserver agreement was 0.69. There was agreement on 19542 $(87 \%)$ of occasions and disagreement on $4715(13 \%)$ of occasions.

The virtual review process identified 13 patients who were deemed high risk by the glaucoma specialist but not by the optometrist; 5 of these patients had been categorised as low risk by the optometrist.

Glaucoma specialists tended to classify patients into less urgent categories reducing the number of review appointments required. Overall, 378 patients who were thought to have glaucoma by the optometrist were said to be normal by the glaucoma specialist and discharged from the system. In all, 3084 patients were classified into less severe categories by the 
A

Single Field Analysis

Eye: Left

Central 24-2 Threshold Test

Fixation Monitor: Blind Spot

Fixation Target: Central

Stimulus: III, White

Fixation Losses: 2/12

Background: 31.5 ASB

False POS Errors: $5 \%$

Strategy: SITA-Fast

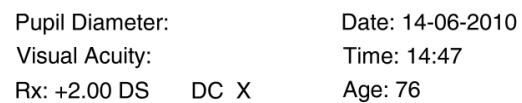

False NEG Errors: $8 \%$

Test Duration: 04:02

Fovea: OFF

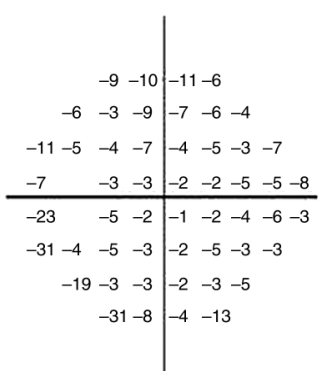

Total Deviation

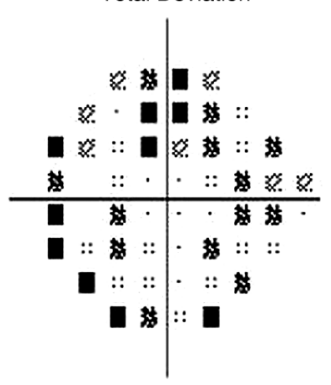

\begin{tabular}{ll|llll}
16 & 16 & -15 & 20 & \\
24 & 19 & 21 & 23 & 23
\end{tabular}

$\begin{array}{llll}6 & 23 & 26 & 23\end{array}\left[\begin{array}{llll}26 & 25 & 26 & 21\end{array}\right.$

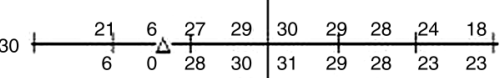

\begin{tabular}{llll|llll}
$<0$ & 26 & 26 & 29 & 29 & 26 & 27 & 25
\end{tabular}

\begin{tabular}{lll|lll}
11 & 27 & 27 & 28 & 27 & 23
\end{tabular}

$<0 \quad 21-25 \quad 16$

Rx. +2.00 DS DC $X \quad$ Age: 76

specialist and 1631 were classified into a more urgent category. The more conservative categorisation created by the glaucoma specialist reduced the number of additional visits that may have been required by 1248 or $2.4 \%$ of the total number of visits (table 2).

\section{DISCUSSION}

Healthcare spending in the UK will fall in real terms over the next several years and glaucoma specialists will need to provide more care with less money. To achieve this and continue to deliver high quality care to glaucoma patients, new models of care will be required. One such model is described herein and uses an internet-based virtual clinic to enable a glaucoma specialist to review all the clinical information and confirm or alter the interim clinical decision made by the assessing optometrist. We analysed the inter-observer agreement between optometrists and glaucoma specialist to determine if this specialist supervision provided an additional benefit to community-based optometrist assessment alone. There was substantial agreement between each group as represented by a Kappa statistic of 0.69 .

Ensuring patient safety is a critical consideration in the development of any new model of care. We assessed patient safety by 
$\mathrm{B}$
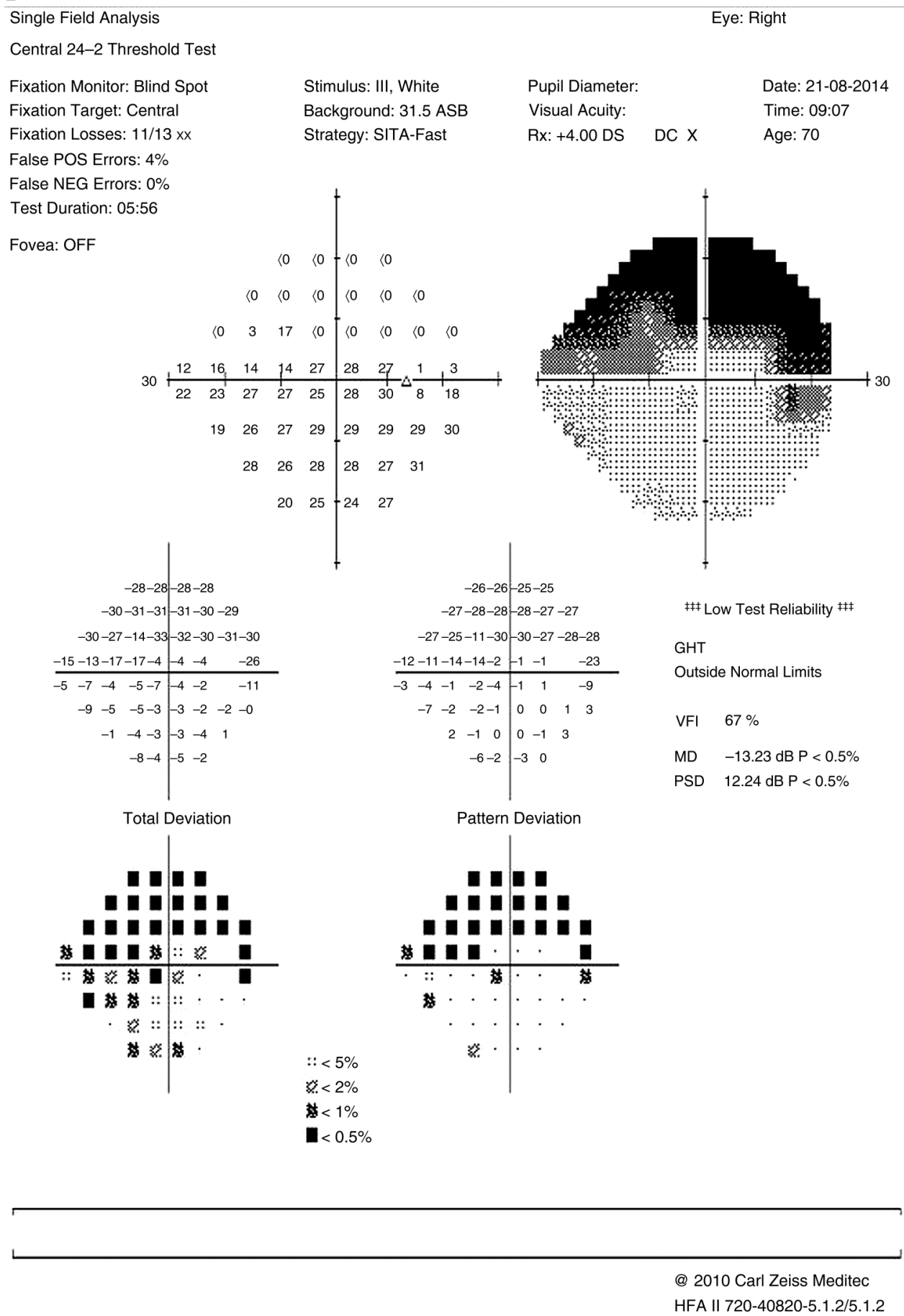

Figure 2 Continued.

determining if any high-risk patients were identified by the glaucoma specialist who had not been identified as high risk by the assessing optometrist. While there were only a small number of high-risk patients missed by the optometrist (13), each of these clinical situations can potentially result in a missed opportunity to prevent blindness and this is a benefit that must not be underestimated, particularly if public confidence in the system is to be maintained. With any novel health intervention, patient safety is paramount; these data demonstrate that consultant supervision catches a relatively large number of patients who were underdiagnosed by the optometrist. In all, 15\% of patients diagnosed as normal by an optometrist were actually found to have glaucoma (94 of 625) and a similar number (13.5\% 691 of 5120 ) of patients who were said to be stable by the optometrist were actually considered to be at higher risk after ophthalmic review. However, potentially the greatest risk to patient safety was the $6.5 \%$ (838 of 12892 ) of patients thought to be low risk by the optometrist who were actually found to be unstable and thus requiring a clinical review within 6 weeks. Delay in these patients seeking a prompt face-to-face consultation with a glaucoma specialist could result in delayed treatment and unnecessary loss of vision.

The purpose of outsourcing is to streamline clinics and maximise the quality of care that can be delivered within an inelastic 
Table 1 Data relating to interim diagnostic category (after the patients had been classified by the optometrist) and final diagnostic category (after the patient data has been re-evaluated by a consultant in the 'virtual clinic')

\begin{tabular}{lrrrrrr}
\hline \multicolumn{7}{l}{ Assigned categories } \\
\hline \multicolumn{5}{l}{ Glaucoma specialist } & & \\
\cline { 2 - 5 } Count & Normal & Stable & Low risk & Unstable & High risk & Total \\
\hline Optometrist & & & & & & \\
$\quad$ Normal & 531 & 49 & 28 & 17 & 0 & 625 \\
Stable & 267 & 4162 & 542 & 149 & 0 & 5120 \\
Low risk & 90 & 1303 & 10661 & 833 & 5 & 12892 \\
Unstable & 21 & 66 & 1334 & 4067 & 8 & 5496 \\
High risk & 0 & 0 & 0 & 3 & 121 & 124 \\
Total & 909 & 5580 & 12565 & 5069 & 134 & 24257 \\
\hline
\end{tabular}

budget. An important part of this involves reviewing patients at intervals appropriate to their risk of blindness. There was an understandable tendency among optometrists to be 'risk-averse' and to review patients more frequently than was thought necessary by glaucoma specialists.

Table 2 shows data pertaining to the total number of annual review appointments required based upon the assumption that 'normal' patients are discharged, that 'stable' patients are seen once per annum, 'low-risk' patients are seen twice per annum and 'unstable' patients seen four times per annum. It can be seen that the virtual review process reduces the overall number of patient review appointments per annum.

By identifying this overservicing and reducing the overall number of reviews by $2.4 \%$, glaucoma specialists created an additional cost saving into the programme. A reduction of $2.4 \%$ in reviews extrapolated to 2.4 million glaucoma attendances throughout England equates to 57600 fewer glaucoma review appointments per year and a saving to the National Health Service (NHS) of nearly $£ 3$ million (assuming a net additional cost of about $£ 50$ per appointment and including cost of the virtual review). However, it must be noted that this study does not include a cost-benefit analysis and no comment can be made about the cost-benefit of a virtual glaucoma clinic compared with traditional inhospital care.

This study has several limitations. It was a retrospective analysis of an operational programme that has been running in the Bristol region and elsewhere for 6 years and therefore selection of patients was not controlled. Data therefore may not be applicable

Table 2 Number of review appointments required per year based upon one appointment per annum for 'stable' patients, two appointments for 'low-risk' patients and four appointments for 'unstable' patients

\begin{tabular}{lcrlrr}
\hline \multicolumn{3}{l}{ Reviews generated } & & & \\
\hline & \multicolumn{2}{l}{ Optometrist } & & & Ophthalmologist \\
\cline { 2 - 3 } Category & Number seen & Reviews & & Number seen & Reviews \\
\hline Normal & 625 & 0 & 909 & 0 \\
Stable & 5120 & 5120 & & 5580 & 5580 \\
Low risk & 12892 & 25784 & & 12565 & 25784 \\
Unstable & 5496 & 21984 & & 5069 & 20276 \\
High risk & 124 & 0 & 134 & 0 \\
Total reviews & & 52888 & & 51640 \\
\hline
\end{tabular}

to other populations. However, it does suggest that virtual clinics conducted over the internet by glaucoma specialists are an important safety oversight where community optometrists may otherwise be managing glaucoma patients in isolation. Furthermore, such virtual clinics potentially cut the cost of delivering glaucoma care in the community by reducing the number of unnecessary review visits. The model described optimises use of resources by using technicians to collect data and optometrists to triage patients before directing only those patients deemed at-risk of visual loss to be seen by specialist medical staff within the hospital. We see this model as an important tool in the delivery of glaucoma care in much the same way that diabetic screening services have evolved to screen for diabetic retinopathy, allowing only those at high risk to be referred for specialist consultation within the HES.

This is the first publication on a novel approach to dealing with the very large number of glaucoma patients that threaten to swamp the NHS. We have suggested that virtual supervision is an appropriate and cost effective part of such a service innovation. Ophthalmic supervision of optometrists provides a useful feedback loop that can assist the optometrist to develop their clinical skills. We have preliminary data that suggest an increase in the kappa score over time as optometrists improve their skills. Further questions need to be addressed in order to maximise cost and resource efficiency. Is the ophthalmic supervision required once optometrists reach a certain level? Are optometrists required or can technicians upload the data for ophthalmic review? Can we use more modern progression analysis software to improve detection of patients who are deteriorating? Can this system be transferred to developing regions where there is a shortage of eye care professionals?

Correction notice This article has been corrected since it was published Online First. A new version of Figure 2 has been used.

Acknowledgements We should like to acknowledge HES managers at Bristol Eye Hospital (Kate Liddington and Geoff Underwood), Kingston Hospital (Nicola Hunt) and George Eliot Hospital, Nuneaton (Heather Norgrove) who have facilitated development of the service.

Contributors HRW contributed to the analysis of data and writing of the paper. JPD contributed to collection and analysis of data and writing of paper.

Competing interests JPD is a director of New Medical Systems (Newmedica). Provenance and peer review Not commissioned; externally peer reviewed.

Open Access This is an Open Access article distributed in accordance with the Creative Commons Attribution Non Commercial (CC BY-NC 4.0) license, which permits others to distribute, remix, adapt, build upon this work non-commercially, and license their derivative works on different terms, provided the original work is properly cited and the use is non-commercial. See: http://creativecommons.org/ licenses/by-nc/4.0/

\section{REFERENCES}

1 NICE: CG85-Glaucoma: diagnosis and management of chronic open angle glaucoma and ocular hypertension. April 2009

2 Kelly S, Rimmer T, Bailey C, et al. New to follow up (N:F) ratios in ophthalmology outpatient services. Royal College of Ophthalmologists Professional Standards Committee V3, 2011.

3 National Patient Safety Agency. Rapid Response Report. Preventing delay to follow up for patients with Glaucoma. 2009.

4 Vernon SA, Adair A. Shared care in glaucoma: a national study of secondary care lead schemes in England. Eye 2010;24:265-9.

5 Spry PGD, Spencer I, Sparrow JM, et al. The Bristol Shared Care Glaucoma Study: reliability of community optometric and hospital eye service test measures. $\mathrm{Br} \mathrm{J}$ Ophthalmol 1999;83:707-12.

6 Banes MJ, Culham LE, Bunce C, et al. Agreement between optometrists and ophthalmologists on clinical management decisions for patients with glaucoma. $\mathrm{Br} J$ Ophthalmol 2006:90:579-85.

7 Azuara-Blanco A, Burr J, Thomas R, et al. The accuracy of accredited glaucoma optometrists in the diagnosis and treatment recommendation for glaucoma. $\mathrm{Br} J$ Ophthalmol 2007;91:1639-43. 